\title{
Class Groups of the Quadratic Fields Found by F. Diaz y Diaz
}

\section{By Daniel Shanks}

\begin{abstract}
F. Diaz y Diaz has discovered 99 discriminants $d$ between -3321607 and -60638515 inclusive for which $Q(\sqrt{d})$ have a 3-rank $r_{3}=3$. These 99 imaginary quadratic fields are analyzed here and the class groups are given and discussed for all those of special interest. In 98 cases, the associated real quadratic fields have $r_{3}=2$, but for $d=44806173=3 \cdot 14935391, Q(\sqrt{d})$ has a class group $C(3) \times C(3) \times C(3)$; and this is now the smallest known $d$ for which a real quadratic field has $r_{3}=3$.
\end{abstract}

By selecting discriminants generated by certain quartic and sextic polynomials, the author and several collaborators [1]-[4] constructed a large number of imaginary quadratic fields having 3-rank $r_{3}$ equal to 3 or 4 . That is, their ideal class groups contain $C(3) \times C(3) \times C(3)$ or $C(3) \times C(3) \times C(3) \times C(3)$ as subgroups. The smallest of these discriminants $d$ was

$$
d=-63199139=-D_{6}(28)
$$

where

$$
D_{6}(z)=108 z^{4}-148 z^{3}+84 z^{2}-24 z+3 .
$$

The smallest class number of these fields, which was $h=1836=27 \cdot 68$, occurred for

$$
d=-3\left(17^{6}+4 \cdot 6^{6}\right) \text {. }
$$

Since the polynomials used were very special, it did seem possible [2, p. 545] that a somewhat smaller $|d|$ than 63199139 might exist having $r_{3}=3$. Again, while an example of $h=27$ for $r_{3}=3$ is most unlikely, it did seem very probable that examples with $h / 27<68$ do exist [2, p. 539]. The reason for the latter is that all of these discriminants $d$, by construction, had $(d / 3)=+1$ or 0 , whereas, to minimize $h$, it is clear that one wants $(d / p)=-1$ for all small primes $p$.

In [5], F. Diaz y Diaz obtained both smaller $|d|$ and smaller $h$ by systematically computing reduced solutions of

$$
4 m^{3}=x^{2}+y^{2} D
$$

for fundamental discriminants $-D$. Sorting these on $D$, one then has $Q(\sqrt{-D})$ with $r_{3} \geqslant 3$ if (1) has 5 or more inequivalent solutions. He thus found 99 values of $D<$ 63199139 with $r_{3}=3$ but none with $r_{3}>3$. His procedure is analogous to that in the 
older work of R. J. Porter [6] , [7], the distinction being that Porter computed irregular determinants $-D$, in Gauss's terminology, with $D$ not necessarily square-free. Porter systematically computed reduced quadratic forms $A u^{2}+2 B u v+C v^{2}$ of order 3 under composition, and then sorted them on their negative determinants $D=A C-B^{2}$.

Of the $99 D$ in [5], the smallest is

$$
D=3321607
$$

while the smallest class number, which is

$$
h=162=27 \cdot 6 \text {, }
$$

occurs for $D=3640387$. By this method, one only obtains $r_{3}$, not the class group, or even the class number. However, Diaz y Diaz included the class groups in [5] for (2), (3) and one other $D$ as follows:

$D$

$$
\begin{array}{ll}
3640387 & C(3) \times C(3) \times C(18) \\
4897363 & C(3) \times C(3) \times C(33) \\
3321607 & C(3) \times C(3) \times C(63) .
\end{array}
$$

Group

For several investigations, such as certain studies of cubic fields (see [8], [9], [10] , [11, pp. 285-286]), it is useful to examine a number of such $D$ and the class groups of their $Q(\sqrt{-D})$. I have examined all $99 D$ in [5] , and in Table 1 I list all 35 of these that have $h \leqslant 27 \cdot 50$. Table 1 lists $D$; its factorization ( $p$ means prime); the ratio $h / 27$; and the class group (with (4) above abbreviated as $3 \times 3 \times 18$, etc.). Also listed is the Dirichlet function $L(1, \chi)=\pi h / \sqrt{D}$, and each field is assigned an identification number \#. Note that (4), (5), and (6) above are \#'s 1, 2, and 10, respectively. Table 2 includes eight other fields selected from the remaining 64 because they are of special interest.

Commentary on These Fields. Field \# 1 has the smallest $h$, the smallest $L(1, \chi)$, and its exponent 18 is minimal although it is duplicated in $\# 3$ and $\# 14$. The 18 th power of each of its integral ideals is principal, cf. [12], [13]. The next smallest exponent is 24 and occurs in \#22 and \#42. $D$ is minimized in \#10, and this is also the smallest prime $D$. But note that in \#5 $Q\left(\sqrt{-1204729)}\right.$ also has $r_{3}=3$, so -1204729 is the smallest prime determinant in Gauss's terminology. The smallest determinant is -1118090 and is found in \#29. Surprisingly, its class group contains $C(6) \times C(6) \times$ $C(6)$. Accordingly,

$$
A^{6}=B^{2}+C^{2} 1118090
$$

has an exceptionally large number of small solutions $A$ such as

\begin{tabular}{rcc}
\multicolumn{1}{c}{$A$} & $B$ & $C$ \\
87 & 459587 & 446 \\
99 & 746681 & 586 \\
117 & 483526 & 1444 , etc.
\end{tabular}

Fields \# 14, 39, 40, 42, and 43 also contain $C(6) \times C(6) \times C(6)$. 
TABLE 1

\begin{tabular}{|c|c|c|c|c|c|}
\hline$D$ & factorization & $h / 27$ & group & $L(1, \chi)$ & \# \\
\hline 3640387 & $421 \cdot 8647$ & 6 & $3 \times 3 \times 18$ & 0.26674 & 1 \\
\hline 4897363 & $p$ & 11 & $3 \times 3 \times 33$ & 0.42162 & 2 \\
\hline 5048347 & $89 \cdot 131 \cdot 433$ & 12 & $3 \times 6 \times 18$ & 0.45302 & 3 \\
\hline 15476323 & $37 \cdot 418279$ & 14 & $3 \times 3 \times 42$ & 0.30186 & 4 \\
\hline 4818916 & $4 \cdot 1204729$ & 16 & $3 \times 3 \times 48$ & 0.61824 & 5 \\
\hline 8992363 & $71 \cdot 126653$ & 16 & $3 \times 3 \times 48$ & 0.45258 & 6 \\
\hline 9778603 & $263 \cdot 37181$ & 16 & $3 \times 3 \times 48$ & 0.43401 & 7 \\
\hline 28114627 & $191 \cdot 147197$ & 18 & $3 \times 3 \times 54$ & 0.28795 & 8 \\
\hline 25012003 & $p$ & 19 & $3 \times 3 \times 57$ & 0.32225 & 9 \\
\hline 3321607 & $p$ & 21 & $3 \times 3 \times 63$ & 0.97737 & 10 \\
\hline 5067967 & $p$ & 23 & $3 \times 3 \times 69$ & 0.86661 & 11 \\
\hline 5288968 & $8 \cdot 661121$ & 24 & $3 \times 3 \times 72$ & 0.88520 & 12 \\
\hline 19941763 & $29 \cdot 687647$ & 24 & $3 \times 3 \times 72$ & 0.45587 & 13 \\
\hline 26156083 & $23 \cdot 43 \cdot 53 \cdot 499$ & 24 & $6 \times 6 \times 18$ & 0.39805 & 14 \\
\hline 42895603 & $53 \cdot 73 \cdot 11087$ & 24 & $3 \times 6 \times 36$ & 0.31083 & 15 \\
\hline 29482627 & $p$ & 25 & $3 \times 3 \times 75$ & 0.39054 & 16 \\
\hline 17496643 & $47 \cdot 372269$ & 26 & $3 \times 3 \times 78$ & 0.52724 & 17 \\
\hline 16006307 & $157 \cdot 269 \cdot 379$ & 28 & $3 \times 6 \times 42$ & 0.59364 & 18 \\
\hline 16784851 & $59 \cdot 284489$ & 30 & $3 \times 3 \times 90$ & 0.62112 & 19 \\
\hline 11324296 & $8 \cdot 359 \cdot 3943$ & 32 & $3 \times 6 \times 48$ & 0.80660 & 20 \\
\hline 35269627 & $241 \cdot 146347$ & 32 & $3 \times 3 \times 96$ & 0.45705 & 21 \\
\hline 55458643 & $29 \cdot 59 \cdot 32413$ & 32 & $3 \times 12 \times 24$ & 0.36448 & 22 \\
\hline 7016747 & $p$ & 33 & $3 \times 3 \times 99$ & 1.05672 & 23 \\
\hline 10348907 & $p$ & 35 & $3 \times 3 \times 105$ & 0.92286 & 24 \\
\hline 10676983 & $p$ & 35 & $3 \times 3 \times 105$ & 0.90857 & 25 \\
\hline 36323563 & $p$ & 35 & $3 \times 3 \times 105$ & 0.49259 & 26 \\
\hline 12201979 & $983 \cdot 12413$ & 36 & $3 \times 3 \times 108$ & 0.87418 & 27 \\
\hline 36399667 & $47 \cdot 137 \cdot 5653$ & 36 & $3 \times 6 \times 54$ & 0.50614 & 28 \\
\hline 4472360 & $8 \cdot 5 \cdot 17 \cdot 6577$ & 40 & $6 \times 6 \times 30$ & 1.60437 & 29 \\
\hline 7060148 & $4 \cdot 109 \cdot 16193$ & 40 & $3 \times 6 \times 60$ & 1.27693 & 30 \\
\hline 19969763 & $11 \cdot 1109 \cdot 1637$ & 40 & $3 \times 6 \times 60$ & 0.75925 & 31 \\
\hline 6562327 & $367 \cdot 17881$ & 42 & $3 \times 3 \times 126$ & 1.39070 & 32 \\
\hline 30580763 & $347 \cdot 88129$ & 42 & $3 \times 3 \times 126$ & 0.64423 & 33 \\
\hline 30470603 & $p$ & 49 & $3 \times 3 \times 147$ & 0.75296 & 34 \\
\hline 54433787 & $613 \cdot 88799$ & 50 & $3 \times 15 \times 30$ & 0.57484 & 35 \\
\hline
\end{tabular}

Note that \#22 contains $C(4) \times C(8)$ and \#35 contains $C(5) \times C(5)$ so their principal genera are irregular not only in their 3-Sylow subgroup but in an additional $p$ Sylow subgroup besides. Field \#36 is the third smallest $D$ but has a relatively large $h$. Field \#37 has the largest $L(1, \chi)$; one finds that its $(d / p)=+1$ for all $p \leqslant 19$. Field 
TABLE 2

\begin{tabular}{rlllll}
\multicolumn{1}{c}{$D$} & \multicolumn{1}{c}{ factorization } & $h / 27$ & group & $L(1, \chi)$ & $\#$ \\
4019207 & $p$ & 69 & $3 \times 3 \times 207$ & 2.91939 & 36 \\
16434239 & $587 \cdot 27997$ & 282 & $3 \times 3 \times 846$ & 5.90049 & 37 \\
21658407 & $3 \cdot 7219469$ & 82 & $3 \times 3 \times 246$ & 1.49456 & 38 \\
24952655 & $5 \cdot 7 \cdot 13 \cdot 173 \cdot 317$ & 208 & $2 \times 6 \times 6 \times 78$ & 3.53198 & 39 \\
28732623 & $3 \cdot 113 \cdot 131 \cdot 647$ & 112 & $6 \times 6 \times 84$ & 1.77233 & 40 \\
34394964 & $4 \cdot 3 \cdot 2866247$ & 80 & $3 \times 6 \times 120$ & 1.15706 & 41 \\
42132596 & $4 \cdot 11 \cdot 17 \cdot 23 \cdot 31 \cdot 79$ & 128 & $2 \times 2 \times 6 \times 6 \times 24$ & 1.67269 & 42 \\
55247159 & $11 \cdot 71 \cdot 127 \cdot 557$ & 288 & $6 \times 6 \times 216$ & 3.28663 & 43
\end{tabular}

\#42 is truly remarkable: it has 6 ramifying primes but none greater than 79 , and an exponent of 24 in spite of its relatively large $D$. It will have an infinite class tower because of its 2 -rank $=5$, cf. [3, p. 187].

Finally, we note that \#38, 40, and 41 are the only three among the $99 D$ in [5] where $D$ is divisible by 3 . The associated real fields $Q(\sqrt{D / 3})$ must, therefore, have $r_{3}=2$ or 3 , cf. [1]. Here, $r_{3}=2$ in all 3 examples; in fact, the class groups are

$\begin{array}{ccc}\# & \text { real field } & \text { group } \\ 38 & Q(\sqrt{9219469}) & 3 \times 3 \\ 40 & Q(\sqrt{9577541}) & 3 \times 12 \\ 41 & Q(\sqrt{2866247}) & 3 \times 15\end{array}$

The real fields for \#38 and \#41 may have the smallest discriminant and determinant, respectively, having $r_{3}=2$ and an associated imaginary field with $r_{3}=3$.

The $99 D$ in [5] are distributed $(\bmod 9)$ in the following interesting way:

\begin{tabular}{l|lllllllll}
$D(\bmod 9)$ & 1 & 2 & 3 & 4 & 5 & 6 & 7 & 8 \\
\hline no. examples & 4 & 4 & 0 & 42 & 43 & 3 & 0 & 3
\end{tabular}

We note, in passing, that $D \equiv 3$ does exist; in fact,

$$
D=3\left(3^{6}+4 \cdot 19^{6}\right) \equiv 3(\bmod 9)
$$

was actually the first known case [1] where $Q(\sqrt{-D})$ had $r_{3}=3$. It is unknown to the author whether an example of $D \equiv 7$ exists.

A Smaller Real Field with $r_{3}=3$. The $96 D$ not divisible by 3 will have associated real fields $Q(\sqrt{3 D})$ with $r_{3}=2$ or 3 . A very simple criterion can decide this immediately for all $89 D \equiv 2,4$, or 5 . It may be deduced from Scholz's second criterion [14] by much the same argumentation as in [2, p. 549], and so we state it without proof:

THEOREM. The 3-rank of $Q(\sqrt{3 D})$ will be 1 less than that of $Q(\sqrt{-D})$ if $D \equiv$ 4 or $5(\bmod 9)$ and $(1)$ has a solution with

$$
y \equiv 1, x \neq 0 \quad(\bmod 9) .
$$


This is also true if $D \equiv 2$ has any solution $(1)$ with $y \equiv 1(\bmod 9)$.

For every $D \equiv 2,4$, and 5 in [5], the solutions of (1) given there readily yield such examples with $y=1$ for one of the smallest values of $m$. Therefore, all of their $Q(\sqrt{3 D})$ also have $r_{3}=2$. By more subtle computations, the remaining $D$, those congruent to 1 or 8 , also have $Q(\sqrt{3 D})$ with $r_{3}=2$ with one single exception for $D \equiv 8$. This exception we list as field \#44:

$\begin{array}{cccccr}D & \text { factorization } & h / 27 & \text { group } & L(1, \chi) & \# \\ 14935391 & p & 135 & 3 \times 3 \times 405 & 2.96305 & 44\end{array}$

We thus discover that, for

$$
d=44806173=3 \cdot 14935391,
$$

the real field $Q(\sqrt{d})$ has $r_{3}=3$.

The principal reduced quadratic form of discriminant (7) is

$$
u^{2}+6693 u v-2481 v^{2} \text {. }
$$

Under the unimodular transformation

$$
\left(\begin{array}{cc}
43 & 96 \\
116 & 259
\end{array}\right)
$$

(8) becomes another reduced form

$$
f(U, V)=2197 U^{2}+3117 U V-3993 V^{2},
$$

and one finds that

$$
f(1,0)=13^{3}, \quad f(1,-1)=(-17)^{3}, \quad f(0,1)=3(-11)^{3} .
$$

But the ideal class number is odd, since $d / 3$ is prime, and so the ramified 3 is principal. Therefore, $(8)$ represents the three cubes: $13^{3},(-17)^{3},(-11)^{3}$. Since the period of reduced forms is only twenty-six forms long until we reach its symmetric midpoint: $(-827,6693,3)$, we readily verify that $(8)$ does not represent

$$
13,-17,-11,-13 \cdot 17,-11 \cdot 13,11 \cdot 17 \text {, or } 11 \cdot 13 \cdot 17 \text {, }
$$

since none of these numbers equals an end-coefficient in a reduced form. As in [1, p. 77], one thus finds that

$$
G=C(3) \times C(3) \times C(3)
$$

is a subgroup of the class group. In fact, as in the earlier case [1], where

$$
d=3^{6}+4 \cdot 19^{6}=188184253,
$$

$G$ is the entire ideal class group. The new $d$ in (7) is smaller; in fact, it is also smaller than the

$$
d=21^{6}+4 \cdot 8^{6}=86814697
$$

of $\left[2\right.$, p. 539], and so it gives the smallest known real $Q(\sqrt{d})$ with $r_{3}=3$. The new 
$Q(\sqrt{d})$ also differs from that with (10) in that its fundamental unit now has norm +1 . Correspondingly, there will be thirteen distinct totally real cubic fields of discriminant $d=44806173$, and that is the smallest known such $d$. The thirteen cubic polynomials are readily obtained from the thirteen solutions (1) with $D=14935391$, cf. [10] .

Computation and Mathematics Department Naval Ship Research and Development Center Bethesda, Maryland 20084

1. DANIEL SHANKS \& PETER WEINBERGER, "A quadratic field of prime discriminant requiring three generators for its class group, and related theory," Acta Arith., v. 21, 1972, pp. 71 87. MR 46 \#9003.

2. DANIEL SHANKS, "New types of quadratic fields having three invariants divisible by 3," J. Number Theory, v. 4, 1972, pp. 537-556. MR 47 \#1775.

3. DANIEL SHANKS \& RICHARD SERAFIN, "Quadratic fields with four invariants divisible by 3," Math. Comp., v. 27, 1973, pp. 183-187; “Corrigenda," ibid., p. 1012 . MR 48 \#8436a, b.

4. CAROL NEILD \& DANIEL SHANKS, "On the 3-rank of quadratic fields and the Euler product," Math. Comp., v. 28, 1974, pp. 279-291.

5. F. DIAZ Y DIAZ, "Sur les corps quadratiques imaginaires dont le 3-rang du groupe des classes est supérieur à 1", Séminaire Delange-Pisot-Poitou, 1973/74, no. G15.

6. R. J. PORTER, “On irregular negative determinants of exponent $9 n$," $M T A C$, v. 10, 1956, pp. 22-25. MR 17, 1140.

7. R. J. PORTER, Tables in the UMT file, MTAC, v. 7, 1953, p. 34; v. 8, 1954, pp. 9697; v. 9, 1955, p. 26, p. 126, p. 198; v. 11, 1957, p. 275 ; v. 12, 1958, p. 225.

8. T. CALlAHAN, "The 3-class groups of non-Galois cubic fields. I," Mathematika, v. 21 , 1974, pp. 72-89.

9. T. CALLAHAN, "The 3-class groups of non-Galois cubic fields. II," Mathematika, v. 21, 1974, pp. $168-188$.

10. DANIEL SHANKS, "Review of Angell's table," Math. Comp., v. 29, 1975, pp. 661665.

11. DANIEL SHANKS, "Calculation and applications of Epstein zeta functions," Math. Comp., v. 29, 1975, pp. $271-287$.

12. DAVID W. BOYD \& H. KISILEVSKY, "On the exponent of the ideal class groups of complex quadratic fields," Proc. Amer. Math. Soc., v. 31, 1972, pp. 433-436. MR 44 \#6644.

13. P. J. WEINBERGER, "Exponents of the class groups of complex quadratic fields," Acta Arith., v. 22, 1973, pp. 117-124. MR 47 \#1776.

14. A. SCHOLZ, "Über die Beziehung der Klassenzahlen quadratischer Körper zueinander," Crelle's J., v. 166, 1932, pp. 201-203. 